\title{
Retinamorphic bichromatic Schrödinger metamedia
}

\author{
V. Labunets ${ }^{1}$, I. Artemov ${ }^{1}$, V. Chasovskikh ${ }^{1}$, E. Ostheimer ${ }^{2}$ \\ ${ }^{1}$ Ural State Forest Engineering University, 620100, Ekaterinburg, Russia \\ ${ }^{2}$ Capricat LLC, Pompano Beach, Florida, USA
}

\begin{abstract}
In this work, we apply quantum cellular automata (QCA) to study pattern formation and image processing in quantum-diffusion Schrödinger metamedia with generalized complex diffusion coefficients. Generalized complex numbers have the real part and imaginary part with the imaginary unit $i^{2}=-1$ (classical case), $i^{2}=+1$ (double numbers) and $i^{2}=0$ (dual numbers). They form three 2-D complex algebras. Discretization of the Schrödinger equation gives the quantum Schrödinger cellular automaton with various complex-valued physical parameters. The process of excitation in these media is described by the Schrödinger equations with the wave functions that have values in algebras of the generalized complex numbers. This medium can be used for creation of the eye-prosthesis (so called the "silicon eye"). The medium suggested can serve as the prosthesis prototype for perception of the bichromatic images.
\end{abstract}

Keywords: Schrödinger equation; Schrödinger transform of image; quantum metamedia; quantum cellular automata; silicon eye; quantum image processing

\section{Introduction}

The metamedia (metamaterials), in which the electro dynamical, thermal and other physical parameters have "exotic" values (negative, imaginary, complex or quaternion ones), shows us the wonderful diversity of dynamic behavior and self-organization types. It is becoming more and clearer that such systems are not exclusive: when researchers try to investigate the nature of complex systems - chemical, biological or physical, - they find many of certain examples. In particular, this fact mainly refers to biological systems, because these systems are always quite far from stable state and their parameters frequently have exotic values. A theoretical quantum brain model was proposed in [1] using a linear and nonlinear Schrödinger wave equation. The model proposes that there exists a quantum process (quantum part of the brain) that mediates the collective response of a neural lattice (classical part of the brain). Perception, emotion etc. are supposed to be emergent properties of such compound a (classical-quantum) neural circuits.

Linear and nonlinear Schrodinger equations [2,3] are important members of the family of methods for image processing, computer vision, and computer graphics. Schrödinger transform of image as a new tool for image analysis was first given in [3]. In the paper, exterior and interior of objects are obtained from Schrödinger transforms of original image and its inverse image. Neural networks and cellular automata (in form of a media) which are compatible with the theory of quantum mechanics and demonstrate the particle-wave nature of information have been analyzed in [4-6]. The studying of processes in such metamedia is very important for many branches of the system theory. There is no general theory of the metamedia yet, and every particular example of similar media, usually provides us with the examples of new dynamic or self-organization types.

In this work, we apply quantum cellular automata to study pattern formation and image processing in quantum-diffusion Schrodinger metamedia with generalized complex diffusion coefficients. Generalized complex numbers have the real part and imaginary part with the imaginary unit $i^{2}=-1$ (classical case), $i^{2}=+1$ (double numbers) and $i^{2}=0$ (dual numbers). They form three 2-D complex algebras. Discretization of the Schrödinger equation gives the quantum Schrödinger cellular automaton with various complex-valued physical parameters. The process of excitation in these media is described by the Schrodinger equations with the wave functions that have values in algebras of the generalized complex numbers. This medium can be used for creation of the eye-prosthesis (so called the "silicon eye"). The medium suggested can serve as the prosthesis prototype for perception of the bichromatic images.

The rest of the paper is organized as follows: in Section 2, the object of the study (the Schrödinger equation) is described. In Section 3, a brief introduction to mathematical background (algebra $\mathrm{A}_{2}(\mathbf{R} \mid i), i^{2}=1,0$ of generalized complex numbers $\mathbf{z}=a+i b$ ) is given (subsection 3.1) in order to understand the concept behind the proposed method. In subsection 3.2, the proposed method based on Schrödinger equations is explained. Next, we defined Schrödinger transform of image, discussed its properties and the properties of the Schrödinger transforms are analyzed. In Section 4, the basic metamedia (the SchrödingerEuclidean, Schrödinger-Minkowskian, Schrödinger-Galilean, Schrödinger-Yaglom) are devised and analyzed in detail. The simulation result and algorithm complexity are demonstrated too. Finally, we gave our conclusion in Section 5.

\section{The object of the study}

In this work the new metamedia with a complex diffusion coefficients are studied. We call such media the Schrödinger metamedia. Classical 2-D heat equation is: 


$$
\frac{\partial \varphi(x, y, t)}{\partial t}=D\left(\frac{\partial^{2} \varphi(x, y, t)}{\partial x^{2}}+\frac{\partial^{2} \varphi(x, y, t)}{\partial y^{2}}\right)+f(x, y, t),
$$

where $\varphi(x, y, t)$ is a function describing the media's excitement, $f(x, y, t)$ is an exciting source (input signal) and $D$ is a diffusion coefficient (real number).

The main purpose of this work is the investigation of derivative laws for Schrödinger metamedia with generalized complex diffusion coefficient in the form of quantum cellular automata. The generalized complex numbers [7] consist of a real part, an imaginary part and a generalized imaginary unit that have one of the following properties: $i_{-}^{2}=-1\left(\mathrm{a}\right.$ classical case), $i_{+}^{2}=+1$ (double numbers) and $i_{0}^{2}=0$ (dual numbers). They form three 2-D complex algebras $\mathrm{A}_{2}(\mathbf{R} \mid i):=\{\mathbf{z}=a+i b \mid a, b \in \mathbf{R}\}$, where $i=i_{-}, i_{0}, i_{+}$. There is a specific type of excitable metamedium for each kind of complex numbers: for $\mathrm{A}_{2}\left(\mathbf{R} \mid i_{-}\right)-$the Schrödinger-Euclidian metamedium (when $\mathbf{D}=D_{c l}+i_{-} D_{q u}$ ), for $\mathrm{A}_{2}\left(\mathbf{R} \mid i_{0}\right.$ ) - the Schrödinger-Galilean metamedium (when $\left.\mathbf{D}=D_{c l}+i_{0} D_{q u}\right)$ and for $\mathrm{A}_{2}\left(\mathbf{R} \mid i_{+}\right)$- the Schrödinger-Minkowskian metamedium (when $\left.\mathbf{D}=D_{c l}+i_{+} D_{q u}\right)$, where $D_{c l}, i D_{q u}$ are classical and quantum diffusion coefficients, respectively.

Excitation of waves in metamedia are described by three Schrödinger equations with a $\mathrm{A}_{2}(\mathbf{R} \mid i)$-valued wave functions $\varphi(x, y, t)$. The discretization of the Schrödinger equations gives us a metamedia models in the form of three excitable cellular automata. Their microelectronic realizations appear to be a programmable Schrodinger metamedia [8].

In this work, we study properties of the Schrödinger excitable metamedium in the form of a cellular automaton. The more detailed information about cellular automata can be found in [9]. The automaton's cells are located inside a 2D array. They can perform basic operations with complex numbers (in different complex algebras $\mathrm{A}_{2}(\mathbf{R} \mid i)$ ). These cells are able to inform the neighboring cells about their states. Such media possess large opportunities in processing of bichromatic images in comparison with the ordinary diffusion media with the real-valued diffusion coefficients. The latter media are used for creation of the eyeprosthesis (so called the "silicon eye"). The medium suggested can serve as the prosthesis prototype for perception of the bichromatic images [10-16].

\section{Methods}

\subsection{Mathematical background}

We consider the algebraic and geometric properties of three 2-D complex algebras $\mathrm{A}_{2}(\mathbf{R} \mid i):=\{\mathbf{z}=x+i y \mid x, y \in \mathbf{R}\}$, where $i=i_{-}, i_{0}, i_{+}$. Additions for all three algebra are identical: $\mathbf{z}_{1}+\mathbf{z}_{2}=\left(x_{1}+i y_{1}\right)+\left(x_{2}+i y_{2}\right)=\left(x_{1}+x_{2}\right)+i\left(y_{1}+y_{2}\right)$, but multiplications are different [7]:

$$
\mathbf{z}_{1} \mathbf{z}_{2}=\left(x_{1}+i y_{1}\right)\left(x_{2}+i y_{2}\right)=\left\{\begin{aligned}
\left(x_{1} x_{2}-y_{1} y_{2}\right)+i\left(x_{1} y_{2}+x_{1} y_{2}\right), & i^{2}=-1, \\
\left(x_{1} x_{2}+y_{1} y_{2}\right)+i\left(x_{1} y_{2}+x_{1} y_{2}\right), & i^{2}=+1, \\
x_{1} x_{2}+i\left(x_{1} y_{2}+x_{1} y_{2}\right), & i^{2}=0 .
\end{aligned}\right.
$$

The conjugation operation can be defined for $\mathrm{A}_{2}(\mathbf{R} \mid i)$. It maps each number $\mathbf{z}=x+i y$ to the number $\overline{\mathbf{z}}=\overline{x+i y}:=x-i y$. It is possible to define a pseudo norm using conjugation.

Definition 1. The quadratic norm

$$
\|\mathbf{z}\|=\mathbf{z} \overline{\mathbf{z}}=x^{2}-i^{2} y^{2}=\left\{\begin{array}{cl}
x^{2}+y^{2}, & \mathbf{z} \in \mathrm{A}_{2}\left(\mathbf{R} \mid i_{-}\right), \\
x^{2}-y^{2}, & \mathbf{z} \in \mathrm{A}_{2}\left(\mathbf{R} \mid i_{+}\right), \\
x^{2}, & \mathbf{z} \in \mathrm{A}_{2}\left(\mathbf{R} \mid i_{0}\right) .
\end{array}\right.
$$

The conjugation operation can be defined is called the pseudonorm of the number $\mathbf{z}=x+i y$. It is easy to check that $N\left(\mathbf{z}_{1} \mathbf{z}_{2}\right)=N\left(\mathbf{z}_{1}\right) N\left(\mathbf{z}_{2}\right)$

Definition 2. The value of an arithmetical square root of the product of numbers $\mathbf{z} \overline{\mathbf{z}}=N(\mathbf{z})$ is called an absolute value of a generalized complex number $\mathbf{z}$ and can be denoted as norm

$$
|\mathbf{z}|=\sqrt{\mathbf{z} \overline{\mathbf{z}}}=\sqrt{x^{2}-i^{2} y^{2}}=\left\{\begin{array}{cc}
\sqrt{x^{2}+y^{2}}, & \mathbf{z} \in \mathrm{A}_{2}\left(\mathbf{R} \mid i_{-}\right), \\
\sqrt{x^{2}-y^{2}}, & \mathbf{z} \in \mathrm{A}_{2}\left(\mathbf{R} \mid i_{+}\right) \\
|x|, & \mathbf{z} \in \mathrm{A}_{2}\left(\mathbf{R} \mid i_{0}\right)
\end{array}\right.
$$

This absolute value can be interpreted as a distance (elliptic, hyperbolic or parabolic) from origin to the point $\mathbf{z}$. In the first case, the absolute value is called elliptic, in the second case we are dealing with a hyperbolic value (it can also take imaginary values because of the result of subtraction operation $\left.x^{2}-y^{2}\right)$ and in the third case, it is called the parabolic absolute value. The generalized complex planes are turned into a 2-D pseudo metrical space if they are equipped the following pseudo metrics: 


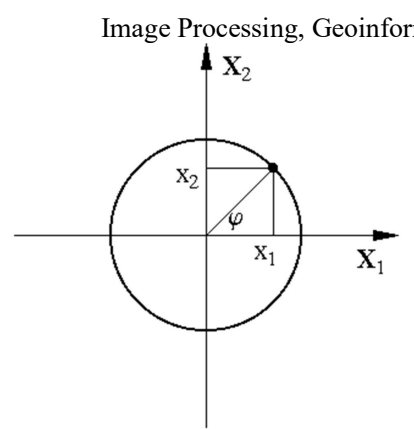

a)

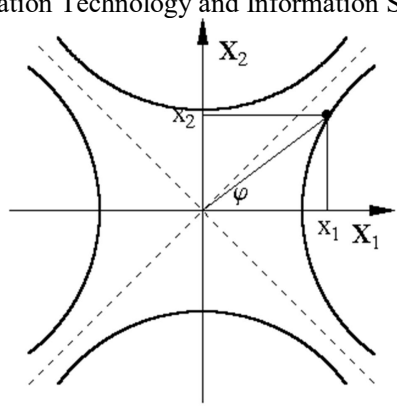

b)

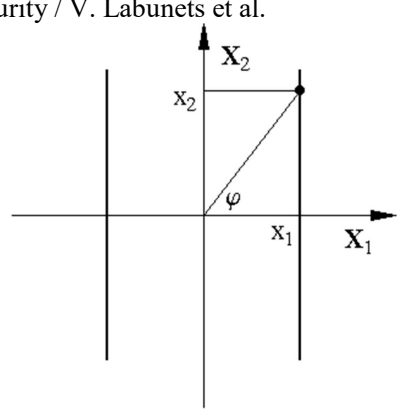

c)

$$
\rho\left(\mathbf{z}_{1}, \mathbf{z}_{2}\right):=\sqrt{\left(\mathbf{z}_{2}-\mathbf{z}_{1}\right) \overline{\left(\mathbf{z}_{2}-\mathbf{z}_{1}\right)}}=\left\{\begin{array}{cc}
\sqrt{\left(x_{2}-x_{1}\right)^{2}+\left(y_{2}-y_{1}\right)^{2}}, & \mathbf{z} \in \mathrm{A}_{2}\left(\mathbf{R} \mid i_{-}\right), \\
\sqrt{\left(x_{2}-x_{1}\right)^{2}-\left(y_{2}-y_{1}\right)^{2}}, & \mathbf{z} \in \mathrm{A}_{2}\left(\mathbf{R} \mid i_{+}\right), \\
\left|x_{2}-x_{1}\right|, & \mathbf{z} \in \mathrm{A}_{2}\left(\mathbf{R} \mid i_{0}\right),
\end{array}\right.
$$

where $\mathbf{z}_{1}=x_{1}+i y_{1}, \mathbf{z}_{2}=x_{2}+i y_{2}$

The algebra $\mathrm{A}_{2}(\mathbf{R} \mid i)$ equipped with pseudo metrics, form three metrical spaces with corresponding geometries: $\mathrm{A}_{2}\left(\mathbf{R} \mid i_{-}\right)$is transformed into the Euclidean geometry, $\mathrm{A}_{2}\left(\mathbf{R} \mid i_{+}\right)$- into the Minkowskian geometry and $\mathrm{A}_{2}\left(\mathbf{R} \mid i_{0}\right)-$ into the Galilean geometry.

Definition 3. The set of all points in the generalized complex plane $\mathrm{A}_{2}(\mathbf{R} \mid i)$ satisfying the equation $|\mathbf{z}|^{2}=x^{2}-i^{2} y^{2}=r^{2}$ is called $\mathrm{A}_{2}(\mathbf{R} \mid i)$-circle of the radius $r$ centered at the origin.

There are three types of circles: $\mathrm{A}_{2}\left(\mathbf{R} \mid i_{0}\right)$-circle is the classical Euclidean (elliptic) circle (Fig.1a), $\mathrm{A}_{2}\left(\mathbf{R} \mid i_{+}\right)$-circle is the Minkowskian (hyperbolic) circle (Fig. $1 \mathrm{~b})$ and $\mathrm{A}_{2}\left(\mathbf{R} \mid i_{0}\right)$-circle is the Galilean (parabolic) circle in the form of two parallel lines (Fig.1c). If $\mathbf{z}=x+i y$ then the generalized complex number $\mathbf{z}_{0}=\mathbf{z} /|\mathbf{z}|$ has the unit modulus if $|\mathbf{z}| \neq 0$. It is easily see, that

$$
\mathbf{z}=|\mathbf{z}|\left(\frac{x}{|\mathbf{z}|}+i \frac{y}{|\mathbf{z}|}\right)=|\mathbf{z}|(\cos \beta+i \cdot \sin \beta),
$$

where

$$
\cos \beta=\frac{x}{\sqrt{x^{2}-i^{2} y^{2}}}=\left\{\begin{array}{ll}
\frac{x}{\sqrt{x^{2}+y^{2}}}=\cos \beta, & \text { if } i=i_{-}, \\
\frac{x}{\sqrt{x^{2}-y^{2}}}=\operatorname{ch} \beta, & \text { if } i=i_{+},
\end{array} \quad \sin \beta=\frac{y}{\sqrt{x^{2}-i^{2} y^{2}}}= \begin{cases}\frac{y}{\sqrt{x^{2}+y^{2}}}=\sin \beta, & \text { if } I=i_{-}, \\
\frac{y}{\sqrt{x^{2}-y^{2}}}= \pm \operatorname{sh} \beta, & \text { if } I=i_{+}, \\
\frac{y}{|x|}=\operatorname{sg} \beta, & \text { if } I=i_{0} .\end{cases}\right.
$$

Here $\cos \beta, \sin \beta$ are generalized trigonometric functions. In the first case ( $i=i_{-}$) generalized trigonometric functions coincide with classical (elliptic) functions: $\cos \beta=\cos \beta, \sin \beta=\sin \beta$. In the second case $\left(i=i_{+}\right)$they are equal to hyperbolic functions $\cos \beta=\operatorname{ch} \beta, \sin \beta=\operatorname{sh} \beta$. The third case $\left(i=i_{0}\right)$ gives us new kinds of trigonometric functions: $\cos \beta=\operatorname{cg} \beta \equiv \pm 1$, $\sin \beta=\operatorname{sg} \beta \equiv \beta$ which will be called parabolic (or Galilean) functions.

According to (4)-(6), an arbitrary generalized complex number with the unit modulus has the following form

$$
\mathbf{z}=e^{i \beta}=(\cos \beta+i \cdot \sin \beta)= \begin{cases}\cos \beta+i_{-} \cdot \sin \beta, & \text { if } i=i_{-}, \\ \operatorname{ch} \beta+i_{+} \cdot \operatorname{sh} \beta, & \text { if } i=i_{+}, \\ \pm 1+i_{0} \cdot \beta, & \text { if } i=i_{0} .\end{cases}
$$

In this work, we study the diffusion equation (or the heat equation) with a diffusion coefficient in the form of a generalized complex number and with $\mathrm{A}_{2}(\mathbf{R} \mid i)$-valued wave function. We will call such equation the generalized Schrodinger equation.

\subsection{The generalized Schrödinger equation and cellular automata}

Consider the following 2-D Schrödinger equation

$$
\frac{d}{d t} \varphi(x, y, t)=\mathbf{D} \cdot\left(\frac{d^{2}}{d x^{2}}+\frac{d^{2}}{d y^{2}}\right) \varphi(x, y, t)+f(x, y, t),
$$

where $\varphi(x, y, t)$ is a wave $\mathrm{A}_{2}(\mathbf{R} \mid i)$-valued function. It describes the state $\varphi(x, y, t)$ (in terms of generalized complex numbers) of a metamedium point with coordinates $(x, y)$ at the moment $t$. In (7) $\mathbf{D}=D_{c l}+i D_{q u}$ is an $\mathrm{A}_{2}(\mathbf{R} \mid i)$-valued diffusion coefficient. If $\mathbf{D} \equiv D_{c l} \in \mathbf{R}$ is a real number then (7) is an ordinary diffusion (or heat) equation in the real ordinary medium (we will call one as the Fourier-Gauss medium). If $\mathbf{D} \equiv i D_{q u} \in \mathbf{C}$ is an imaginary number then (7) becomes an ordinary Schrödinger equation with the Plank's constant $i D_{q u} \in i / 2 m$ If $\mathbf{D}=D_{c l}+i D_{q u}=|\mathbf{D}(\cos \beta+i \sin \beta)=| \mathbf{D} \mid e^{i \beta} \in \mathrm{A}_{2}(\mathbf{R} \mid i)$, then (7) is our 
generalization of both diffusion and Schrodinger equations. In case of zero initial conditions, we can write the solution (7) in the form of the Cauchy integral:

$$
\varphi(x, y, t)=\int_{0}^{t} \frac{1}{(2 \sqrt{\pi \mathbf{D}(t-\tau)})^{2}}\left(\int_{-\infty}^{+\infty} \int_{-\infty}^{+\infty} e^{-\frac{(x-\xi)^{2}+(y-\eta)^{2}}{4 \mathbf{D}(t-\tau)}} f(\xi, \eta, \tau) d \xi d \eta\right) d \tau .
$$

This integral we will call the generalized Schrödinger transform (GST) of the initial image $f(x, y, t)$. If $i D_{q u} \in i / 2 m \in \mathbf{C}=\mathrm{A}_{2}\left(\mathbf{R} \mid i_{-}\right)$, then GST is ordinary Schrödinger transform [2-6].

Let us introduce a 2-D regular lattice with nodes $\left(x_{n}, y_{m}, t_{k}\right)$, where $x_{n+1}=x_{n}+h, \quad y_{m+1}=y_{m}+h$ and $t_{k+1}=t_{k}+\tau$. Here $h$ and $\tau$ are spaces between nodes on the space $\mathbf{Z}_{S p}^{2} \subset \mathbf{R}^{2}$ and time $\mathbf{Z}_{t} \subset \mathbf{R}_{t}$ lattices, respectively. For discrete Laplacian we use the following approximation:

$$
\begin{aligned}
& d^{2} \varphi / d x^{2}=\varphi\left(x_{n}+1, y_{m}, t_{k}\right)+\varphi\left(x_{n}-1, y_{m}, t_{k}\right)-2 \varphi\left(x_{n}, y_{m}, t_{k}\right), \\
& d^{2} \varphi / d y^{2}=\varphi\left(x_{n}, y_{m}+1, t_{k}\right)+\varphi\left(x_{n}, y_{m}-1, t_{k}\right)-2 \varphi\left(x_{n}, y_{m}, t_{k}\right), \\
& d^{2} \varphi / d t=\varphi\left(x_{n}, y_{m}, t_{k}+1\right)-\varphi\left(x_{n}, y_{m}, t_{k}\right) .
\end{aligned}
$$

As a result, we get the 2-D discrete Schrödinger equation

$$
\begin{aligned}
& \varphi\left(x_{n}, y_{m}, t_{k}+1\right)=\varphi\left(x_{n}, y_{m}, t_{k}\right)+ \\
& +\mathbf{D} \cdot\left[\varphi\left(x_{n}+1, y_{m}, t_{k}\right)+\varphi\left(x_{n}-1, y_{m}, t_{k}\right)+\varphi\left(x_{n}, y_{m}+1, t_{k}\right)+\varphi\left(x_{n}, y_{m}-1, t_{k}\right)-4 \varphi\left(x_{n}, y_{m}, t_{k}\right)\right] .
\end{aligned}
$$

Now, we give the definition of a 2-D "cellular space" (2-D regular lattice) in which the cellular automaton is defined. A regular lattice $\mathbf{Z}_{S p}^{2} \subset \mathbf{R}_{S p}^{2}$ consists of a set of cells (elementary automata, or electrical circuits A $u t$ ), which homogeneously cover a 2-D Euclidean space. Each cell is labeled by its position $\operatorname{Au} t\left(x_{n}, y_{m}\right)=\operatorname{Au} t(n, m),(n, m) \in \mathbf{Z}_{S p}^{2}$

Regular, discrete, infinite network consisting of a large number of simple identical elements in the form of elementary automata $\mathrm{A} \boldsymbol{u} t(n, m)$ a copy of which will take place at each node $(n, m)$ of the net is called the cellular automaton (see Fig.2 and Fig.3a). Each so decorated note will be called a cell A wt $(n, m)$ and will communicate with a finite number of other cells A $\boldsymbol{u} t(i, k)$, which determine its neighborhood $(i, k) \in \mathbf{M}(m, n)$, geometrically uniform $\mathbf{M}(m, n) \equiv \mathbf{M}, \forall \mathbf{M}(m, n) \in \mathbf{Z}_{S p}^{2} . \quad T h e$ neighborhood of the cell A $\boldsymbol{u} t(n, m)$ (including the cell itself or not, in accordance with convention) is the set of all the cells A $\boldsymbol{u} t(i, k),(i, k) \in \mathbf{M}(m, n)$ of the network which will locally determine the evolution of A $\boldsymbol{u} t(n, m)$. This local communication, which is deterministic, uniform and synchronous determines a global evolution of the cellular automaton, along discrete time steps $t_{k+1}=t_{k}+\tau$.

In the case of $\mathbf{Z}_{S p}^{2}$, the classical neighborhoods are the von Neumann's and Moore's ones. They are known as the nearest neighbors neighborhoods, and defined according to the usual norms and the associated distances. More precisely, for $(i, j) \in \mathbf{Z}_{S p}^{2},\|(i, j)\|_{1}=|i|+|j|$ and $\|(i, j)\|_{\infty}=\max (|i|,|j|)$ will denote ${ }_{1}$ - and ${ }_{\infty}$-norm respectively. Let $\rho_{1}$ and $\rho_{\infty}$ be the associated distances. Then Von Neumann and Moore neighborhoods (Fig.2) are $\mathbf{M}_{+}(m, n):=\left\{(i, k) \mid \rho_{1}((m, n),(i, k)) \leq 1\right\}$ and $\mathbf{M}(m, n):=\left\{(i, k) \mid \rho_{\infty}((m, n),(i, k)) \leq 1\right\}$, respectively. To each cell Aut $(n, m)$ we assign an $\mathrm{A}_{2}(\mathbf{R} \mid i)$-valued state $\varphi(n, m, k)=\varphi\left(x_{n}, y_{m}, t_{k}\right)$ (i.e., the media's excitement). The dynamics of the cellular automaton are determined by a local transition rule, which specifies the new state $\varphi(n, m, k+1)=\varphi\left(x_{n}, y_{m}, t_{k+1}\right)$ of a cell as a function of its interaction Von Neumann neighborhood configuration, according to (10), i.e.,

$$
\begin{aligned}
& \varphi(n, m, k+1)=\varphi(n, m, k)+ \\
& +\mathbf{D} \cdot[\varphi(n+1, m, k)+\varphi(n-1, m, k)+\varphi(n, m+1, k)+\varphi(n, m-1, k)-4 \varphi(n, m, k)] .
\end{aligned}
$$

This rule shows us the relation between a state $\varphi(n, n, k+1)$ of the cell Aut $(n, m)$ at the current moment time $k+1$ and the state $\varphi(n, m, k)$ the same cell $\operatorname{Aut}(n, m)$ and the states of the four neighboring cells $\varphi(n+1, m, k), \varphi(n-1, m, k)$, $\varphi(n, m+1, k), \varphi(n, m-1, k)$ at the previous moment time $k$.

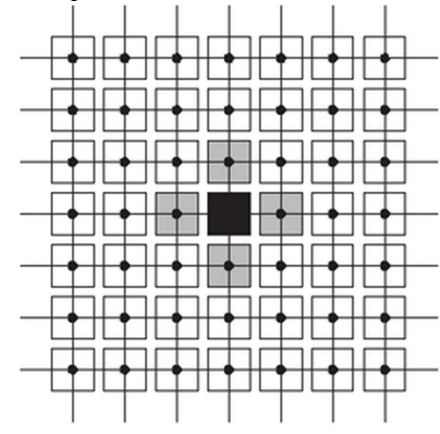

a)

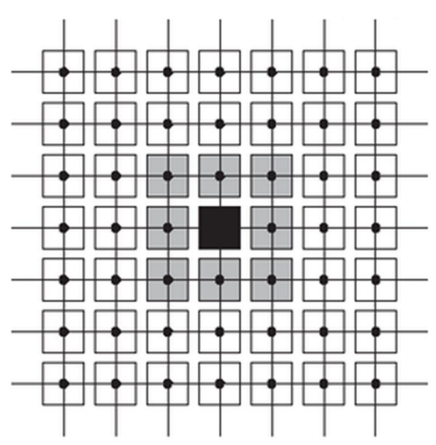

b)

Fig. 2. Examples of interaction neighborhoods (gray and black cells) for the black cell in a 2-D square lattice $\mathbf{Z}_{S p}^{2}$.Von Neumann neighborhoods $\mathbf{M}_{+}(m, n)$ and b) Moore neighborhoods $\mathbf{M}(m, n)$.

The global time evolution of the cellular automaton depends on an algebraic nature of the number $\mathbf{D}$. If it is a real number $\mathbf{D} \equiv D_{c l} \in \mathbf{R}$ then the automaton simulates the heat propagation on a 2-D plane. According to the results of analysis, in this case an elementary medium's cell is an ordinary RC-circuit (see Fig. 3b). It is interesting to investigate the global time evolution of the Schrödinger cellular automaton with diffusion coefficient in the form of a generalized complex number 
$\mathbf{D}=D_{c l}+i D_{q u} \in \mathrm{A}_{2}(\mathbf{R} \mid i)$, where $i^{2}= \pm 1,0$. The analysis shows that in this case the elementary cells of a 2-D Schrödinger cellular automata are not RC-circuits, but a 2-channel filters (see Fig. 3c).

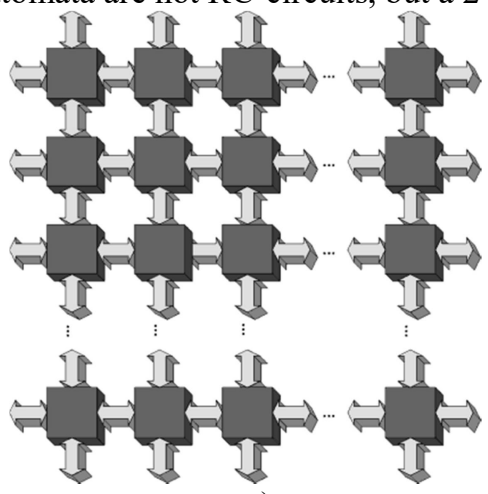

a)

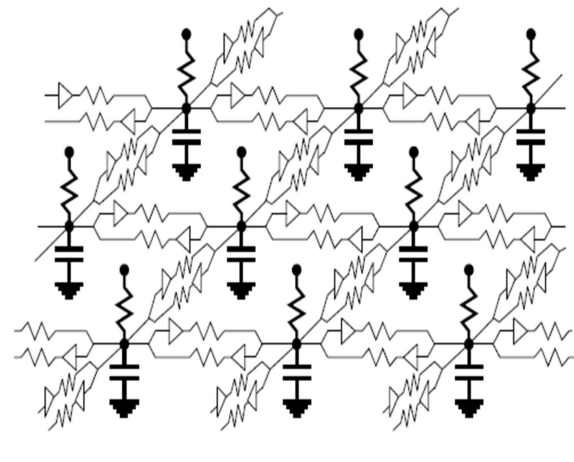

b)

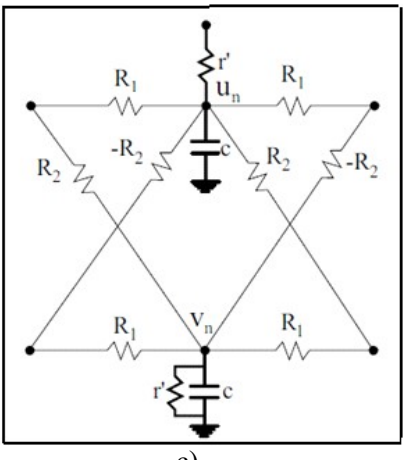

c)

Fig. 3. a) The 2-D cellular automaton (the Schrödinger metamedium) and b) its equivalent electrical circuit in the form of spatially distributed RC-circuit that simulates a simple diffusion media, c) a single cell $\mathrm{A} \boldsymbol{w} t(n, m)$ of the 2-D cellular automaton in form of a 2-channel (complex) filter.

\section{Results and Discussion}

\subsection{The Schrodinger-Euclidean metamedium}

For studying the global time evolution of the Schrödinger cellular automaton we will use the fixed absolute value of $\mathbf{D}$, namely $|\mathbf{D}|=0.11$, which provides quite fast process of diffusion propagation in the classical case with a real-valued diffusion coefficient $\mathbf{D} \equiv D_{0}=0.11$, but will not lead to the memory overflow because of extremely high values

For a classical complex case $\left(i^{2}=-1\right)$, the diffusion coefficient can be represented in the polar form: $\mathbf{D}=D_{c l}+i_{-} D_{q u}=$ $=\sqrt{D_{c l}^{2}+D_{q u}^{2}} \cdot e^{i_{-} \cdot \beta}=D_{0} \cdot e^{i_{-} \cdot \beta}$, where $D_{0}=\sqrt{D_{c l}^{2}+D_{q u}^{2}}, \beta=\operatorname{arctg}\left(D_{q u} / D_{c l}\right)$. An ordinary diffusion occurs when $\beta=0$ (realvalued diffusion coefficient). The quantum diffusion (for free quantum particle) occurs when $\beta=\pi / 2$ (a purely imaginary diffusion coefficient like the one in the Schrodinger equation). It is interesting to study how the global time evolution is changing when the angle $\beta$ runs along interval $0 \leq \beta \leq \pi / 2$. For $\beta=0$ we have the Fourier-Gaussian medium (classical Newton world), and for $\beta=\pi / 2$ we have the Schrödinger-Euclidean medium (quantum world). On the $\beta$ 's increase a classical diffusion Fourier-Gaussian medium turns into the quantum Schrödinger-Euclidean medium.

On Fig. 4 and Fig. 5 the results of modeling for a complex diffusion coefficient $D$ with different values of phase $\beta$ are presented. Each picture is divided onto four parts: the bottom row represents a real $\mathfrak{R}\{\varphi(x, y, t)\}$ and an imaginary $\mathfrak{I}\{\varphi(x, y, t)\}$ components of a wave excitement in the form of $\mathrm{A}_{2}\left(\mathbf{R} \mid i_{-}\right)$-valued function $\varphi(x, y, t)$, the absolute value $|\varphi(x, y, t)|$ is presented in the top left quarter, the phase $\operatorname{Arg}\{\varphi(x, y, t)\}$ is shown in the top right quad.

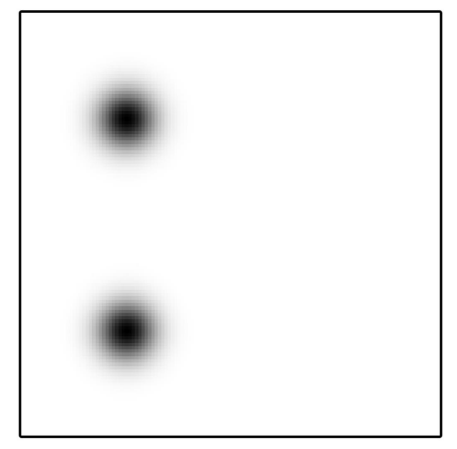

a) $\beta=0^{0}$

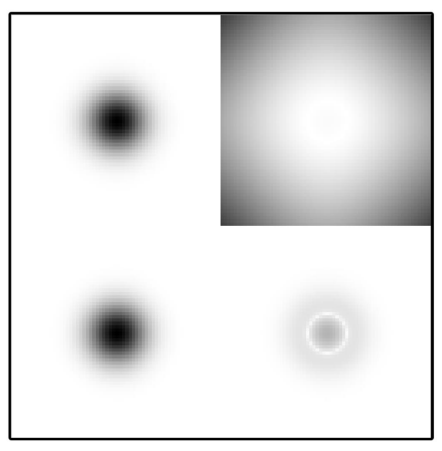

b) $\beta=5^{0}$

Fig. 4. The excitement of the Schrödinger-Euclidean metamedium at the time $t_{k}=128$ for two values of diffusion coefficient $\mathbf{D}=D_{0} \cdot e^{i_{-} \cdot \beta}$, where $\beta=0^{0}$ (the Fourier-Gaussian medium) and $\beta=5^{0}$ (the Schrödinger-Euclidean metamedium).

At the initial moment of time a single cell Aut $\left(x_{0}, y_{0}\right)$ was being excited by the bichromatic Dirac delta-function $\varphi\left(x_{0}, y_{0}, t=0\right)=\left[\delta\left(x_{0}, y_{0}, 0\right)+i \delta\left(x_{0}, y_{0}, 0\right)\right]=\delta\left(x_{0}, y_{0}, 0\right)[1+i]$. In process of time, the excitement covers more and more cells of automaton. Fig. 4 shows the excitement of a metamedium with two diffusion coefficients: $\beta=0^{0}$ (a real-valued diffusion coefficient) and $\beta=5^{0}$ at the moment of time $t_{k}=128$. It can be seen that when $\beta=0^{0}$ the excitement takes the form of the 2D Gaussian surface (see Fig. 4a and Fig. 5a) and describes an ordinary diffusion process. Dark intensities correspond to higher 
number values on the mentioned figures. When $\beta=5^{0}$ the excitement has not very strongly marked form of a blurred wave packet (see Fig. $4 \mathrm{~b}$ and Fig. $5 \mathrm{~b}$ ). The wave nature denotes on the appearance of quantum properties of the Schrodinger-Euclid metamedium even with small values of an angle $\beta$.

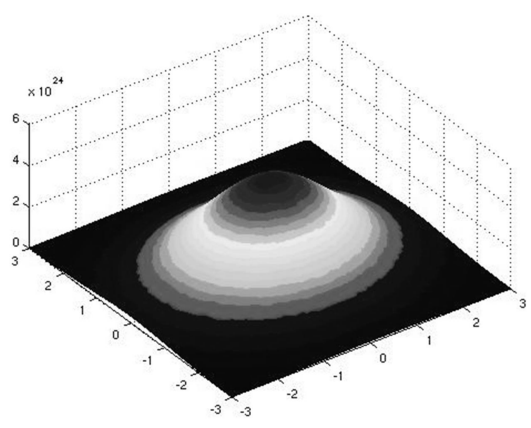

a) $\beta=0^{0}$

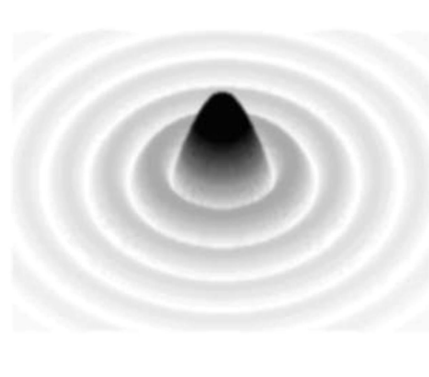

b) $\beta=5^{0}$

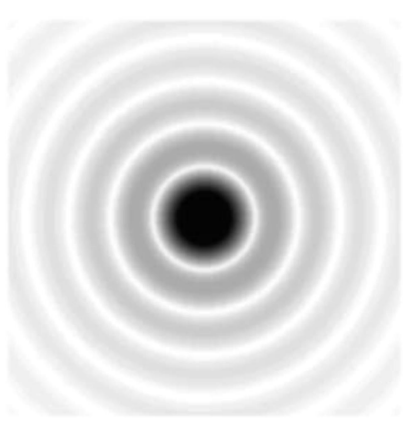

Fig. 5. The typical excitements a) in the form of 2-D Gaussian surface in the Fourier-Gaussian metamedium and b) in the form of a wave packet in the Schrödinger-Euclidean metamedium $\left(t_{k}=128, \beta=5^{0}\right)$.

Remark 1. Note that with small angles $\beta$ the absolute value of an imaginary component is significantly smaller than an absolute value of a real part: $|\mathfrak{I}\{\varphi(x, y, t)\}|<<|\mathfrak{R}\{\varphi(x, y, t)\}|$. For this reason in $|\varphi(x, y, t)|=\sqrt{(\mathfrak{J}\{\varphi(x, y, t)\})^{2}+(\mathfrak{R}\{\varphi(x, y, t)\})^{2}}$ $\approx|\Re\{\varphi(x, y, t)\}|$ the real value prevails. Thereby in this case (low values of $\beta$ ) the real part and the absolute value of an excitement function have the form of a smooth Gaussian surface. For the visualization of low imaginary part, the normalization has been applied on a Fig $4 \mathrm{~b}$ and Fig $5 \mathrm{~b}$.

When the $\beta$ value is being increased, firstly, a real part also begin to demonstrate a wave nature, and secondly the values of an imaginary and a real parts are flattening $|\mathfrak{I}\{\varphi(x, y, t)\}| \approx|\mathfrak{R}\{\varphi(x, y, t)\}|$. It is shown on a Fig. 6 that when the phase $\beta$ is being increased, the excitement becomes more and more similar to a wave packet in form of a 2-D Gaussian surface. Inside of that surface the real and imaginary components are fluctuating in an antiphase (see interchanging black and white rings on a figure). The absolute value of an excitement has the form of a 2-D Gaussian surface.

\subsection{The Schrödinger-Minkowskian metamedium}

In this case the diffusion coefficient is a double number and the wave function $\varphi(x, y, t)$ takes its values in the algebra of double numbers $\mathrm{A}_{2}\left(\mathbf{R} \mid i_{+}\right):=\left\{z=a+i_{+} b \mid a, b \in \mathbf{R}\right\}$ where $i_{+}^{2}=+1$.
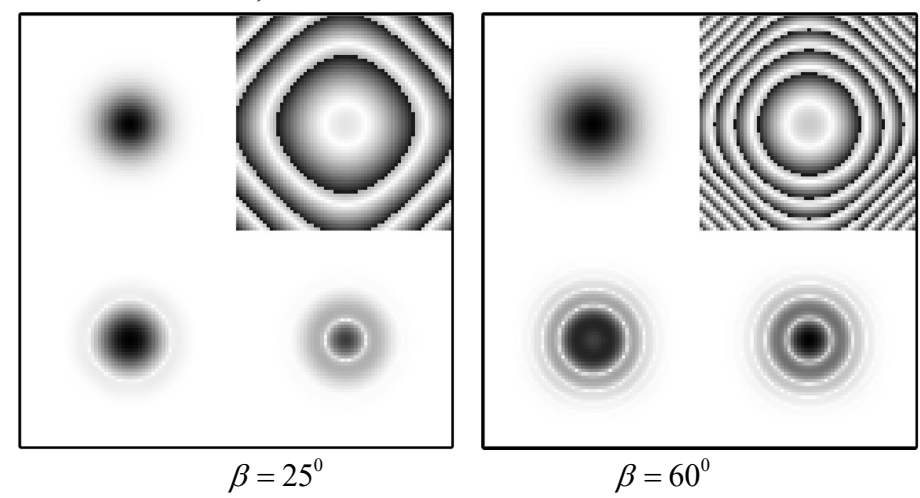

Fig. 6. The excitement of the Schrodinger-Euclidean metamedium at the moment $t_{k}=128$ for two values of diffusion coefficient $\mathbf{D}=D_{0} \cdot e^{i_{-} \cdot \beta}\left(\beta=25^{0}\right.$

$$
\text { and } \beta=60^{\circ} \text { ). }
$$

Every double number can be represented in the following polar form $\mathbf{D}=D_{c l}+i_{+1} D_{q u}=|\mathbf{D}|\left(\cosh \beta+i_{+1} \cdot \sinh \beta\right)=$ $=|\mathbf{D}| e^{i_{+} \cdot \beta} \in \mathrm{A}_{2}\left(\mathbf{R} \mid i_{+1}\right)$, where $|\mathbf{D}|=D_{0}=\sqrt{D_{c l}^{2}-D_{q u}^{2}}, \beta=\operatorname{arcth}\left(b_{0} / a_{0}\right)$. Here we consider the case when $D_{c l}>D_{q u}$. It was done so to get an absolute value $|\mathbf{D}|=\sqrt{D_{c l}^{2}-D_{q u}^{2}}$ that doesn't appear to be a complex number. In addition, the values $D_{c l}^{2}, D_{q u}^{2}$ were chosen so that $|\mathbf{D}|=D_{0}=\sqrt{D_{c l}^{2}-D_{q u}^{2}}=0.11$. Fig. 7 contains the picture of the excitement of a cellular automaton after 128 iterations (initial excitement is the bichromatic Dirac function). 

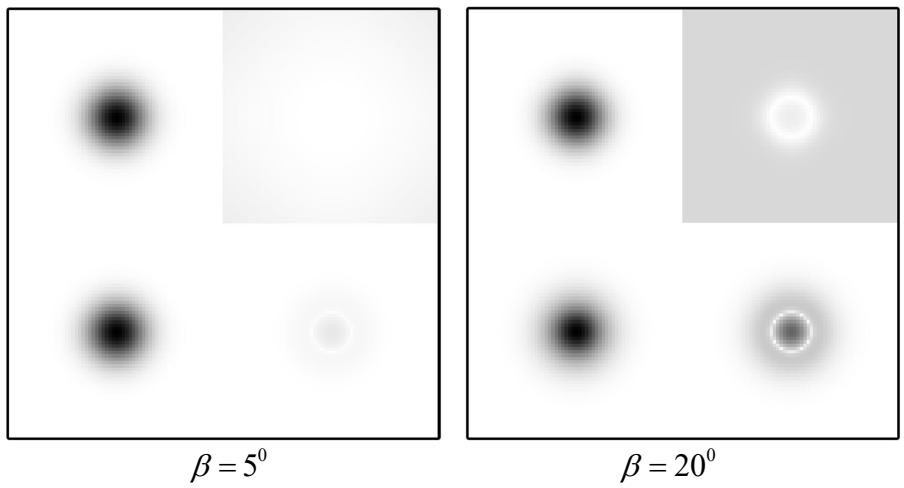

Fig. 7. The excitement of a Schrödinger-Minkowskian metamedium at the moment $t_{k}=128$ for two values of a diffusion coefficient's phase $\mathbf{D}=D_{0} \cdot e^{i_{+} \cdot \beta}$

$$
\left(\beta=5^{0} \text { and } \beta=20^{\circ}\right) \text {. }
$$

Unlike the previous case (when both real and imaginary values had the wave nature) in this case a real component has a smooth Gaussian form and real part has the form of a wave packet. It turned out that the frequency of fluctuations of real values' waves does not increase when the phase of a diffusion coefficient $D$ grows. In the center of a phase image (top right quarter of pictures) the increase of an angle $\beta$ leads to the sharper look of a zero phases ring.

\subsection{The Schrödinger-Galilean metamedium}

In this case the diffusion coefficient $\mathbf{D}$ is a dual number and wave function $\varphi(x, y, t)$ takes its values in the algebra of dual numbers $\mathrm{A}_{2}\left(\mathbf{R} \mid i_{0}\right):=\left\{z=a+i_{0} b \mid a, b \in \mathbf{R}\right\}$, where $i_{0}^{2}=0$. Every dual number can be represented in the following polar form $\mathbf{D}=D_{c l}+i_{0} D_{q u}=|\mathbf{D}|\left( \pm 1+i_{0} \beta\right)=\left|D_{c l}\right| e^{i_{0} \cdot \beta} \in \mathrm{A}_{2}\left(\mathbf{R} \mid i_{0}\right)$, where $|\mathbf{D}|=\left|D_{c l}\right|, \beta=D_{q u} /\left|D_{c l}\right|$. On Fig. 8 we can see the form of excitement process after 128 iterations from the impact of the Dirac delta-function at the initial moment of time.

As in the previous case, a real component does not demonstrate a wave nature when an imaginary component does. What is more, when we increase the value of $\beta$ to $\beta \leq 45^{\circ}$ then the average value of a real part becomes lower than the average value of an imaginary part, and when $\beta>45^{\circ}$ an imaginary component begins to prevail over the real one. In addition, in this case the wave nature of the absolute value of a wave function is absent because of the fact that it does not include a non-zero imaginary part. The reason of this is that for dual numbers we have an equation $|\mathbf{z}|=\left|x+i_{0} y\right|=|x|$. In this way the imaginary part of a wave function is "living on its own", it does not have an impact on an absolute value. So, it is like an invisible "ghost" that accompanies it.
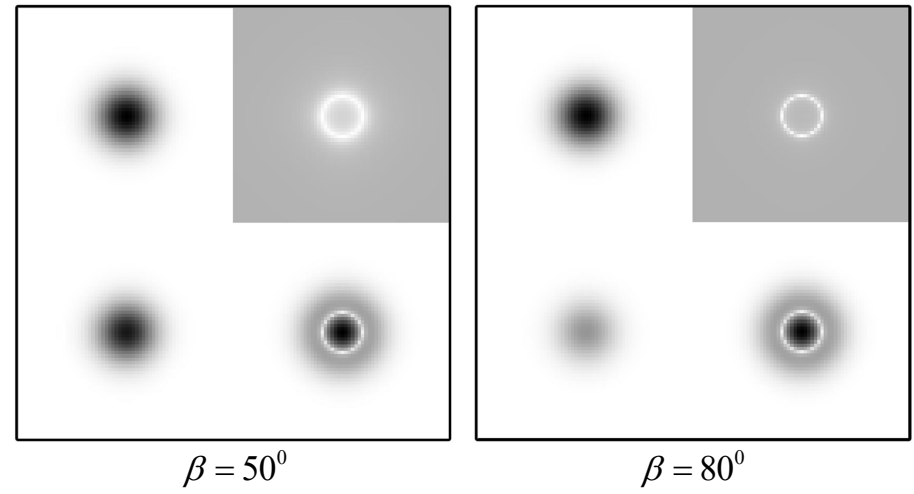

Fig. 8. The excitement of a Schrödinger-Minkowskian metamedium at the moment $t_{k}=128$ for two values of a diffusion coefficient's phase $\mathbf{D}=D_{0} \cdot e^{i_{+} \cdot \beta}$

$$
\left(\beta=5^{0} \text { and } \beta=20^{\circ}\right) \text {. }
$$

\subsection{The Schrödinger-Yaglom metamedium}

The generalization of three algebra $\mathrm{A}_{2}(\mathbf{R} \mid i):=\{\mathbf{z}=a+i b \mid a, b \in \mathbf{R}\}$, where $i=i_{-}, i_{0}, i_{+}$, is the Yaglom algebra [7] $\mathrm{A}_{2}\left(\mathbf{R} \mid i_{k}\right):=\left\{\mathbf{z}=a+i_{k} b \mid a, b \in \mathbf{R}\right\}$ in which we have $i_{k}^{2}=k \in \mathbf{R}$, where $k$ is an arbitrary real number (see Fig. 9). Particularly when $k=1,0$ an algebra $\mathrm{A}_{2}\left(\mathbf{R} \mid i_{k}\right)$ turns into $\mathrm{A}_{2}(\mathbf{R} \mid i)$. In this algebra, the addition and multiplication rules have the following form:

$$
\begin{gathered}
\mathbf{z}_{1}+\mathbf{z}_{2}=\left(x_{1}+i_{k} y_{1}\right)+\left(x_{2}+i_{k} y_{2}\right)=\left(x_{1}+x_{2}\right)+i_{k}\left(y_{1}+y_{2}\right), \\
\mathbf{z}_{1} \mathbf{z}_{2}=\left(x_{1}+i_{k} y_{1}\right)\left(x_{2}+i_{k} y_{2}\right)=\left(x_{1} x_{2}+k \cdot y_{1} y_{2}\right)+i_{k}\left(x_{1} y_{2}+x_{1} y_{2}\right) .
\end{gathered}
$$


The conjugation operation can be defined in the algebra $\mathrm{A}_{2}\left(\mathbf{R} \mid i_{k}\right)$. Such operation maps each number $\mathbf{Z}=x+i_{k} y$ in a new number $\overline{\mathbf{z}}=\overline{x+i_{k} y}:=x-i_{k} y$. It is obvious that $\|\mathbf{z}\|=\mathbf{z} \overline{\mathbf{z}}=x^{2}-k y^{2}$. It can be easily seen that

$$
\mathbf{z}=|\mathbf{z}|\left(\frac{x}{|\mathbf{z}|}+i \frac{y}{|\mathbf{z}|}\right)=|\mathbf{z}| \cdot\left(\frac{x}{x^{2}-k \cdot y^{2}}+i_{k} \cdot \frac{y}{x^{2}-k \cdot y^{2}}\right)=|\mathbf{z}| \cdot\left(\cos _{k} \beta+i_{k} \cdot \sin _{k} \beta\right)=|\mathbf{z}| \cdot e^{i_{k} \beta},
$$

where

$$
\cos _{k} \beta:=\frac{x}{|\mathbf{z}|}=\frac{x}{\sqrt{x^{2}-k y^{2}}}, \quad \sin _{k} \beta:=\frac{y}{|\mathbf{z}|}=\frac{y}{\sqrt{x^{2}-k y^{2}}}, \operatorname{tg}_{k} \beta=\frac{\sin _{k} \beta}{\cos _{k} \beta}=\frac{b}{a} .
$$

In the considered case the diffusion coefficient $D$ is the $\mathrm{A}_{2}\left(\mathbf{R} \mid i_{k}\right)$-valued complex number and the wave function $\varphi(x, y, t)$ takes its values in the algebra $\mathrm{A}_{2}\left(\mathbf{R} \mid i_{k}\right)$, where $i_{k}^{2}=k$. We will call the corresponding medium the Schrodinger-Yaglom metamedium. According to (12) every $\mathrm{A}_{2}\left(\mathbf{R} \mid i_{k}\right)$-valued diffusion coefficient can be represented in a polar form:

$$
\mathbf{D}=|\mathbf{D}|\left(\frac{D_{c l}}{|\mathbf{D}|}+i_{k} \frac{D_{q u}}{|\mathbf{D}|}\right)=|\mathbf{D}| \cdot\left(\frac{D_{c l}}{D_{c l}^{2}-k \cdot D_{q u}^{2}}+i_{k} \cdot \frac{D_{q u}}{D_{c l}^{2}-k \cdot D_{q u}^{2}}\right)=|\mathbf{D}| \cdot\left(\cos _{k} \beta+i_{k} \cdot \sin _{k} \beta\right)=|D| \cdot e^{i_{k} \beta} .
$$

Now $\mathbf{D}$ depends on two parameters $\beta$ and $k$. The results of modeling the Schrödinger-Yaglom metamedia for different values of $k$ are shown on Fig. 10. It should be noted that the ring of zero phases (the bright one), which was inherent for the case with dual numbers $(k=0)$ also is the first inner ring of phase fluctuations for the negative values of a parameter $k$ (on a Fig. $10 k=-0,25$ and $k=-0,05)$. We can see the second bright ring that is located after the first one and also after the first black ring. The second bright ring moves away from the point of origin when the absolute value of $k$ is being decreased. When $|k| \rightarrow 0$ (see Fig. 10c) the mentioned ring along with the first dark one tends to infinity.

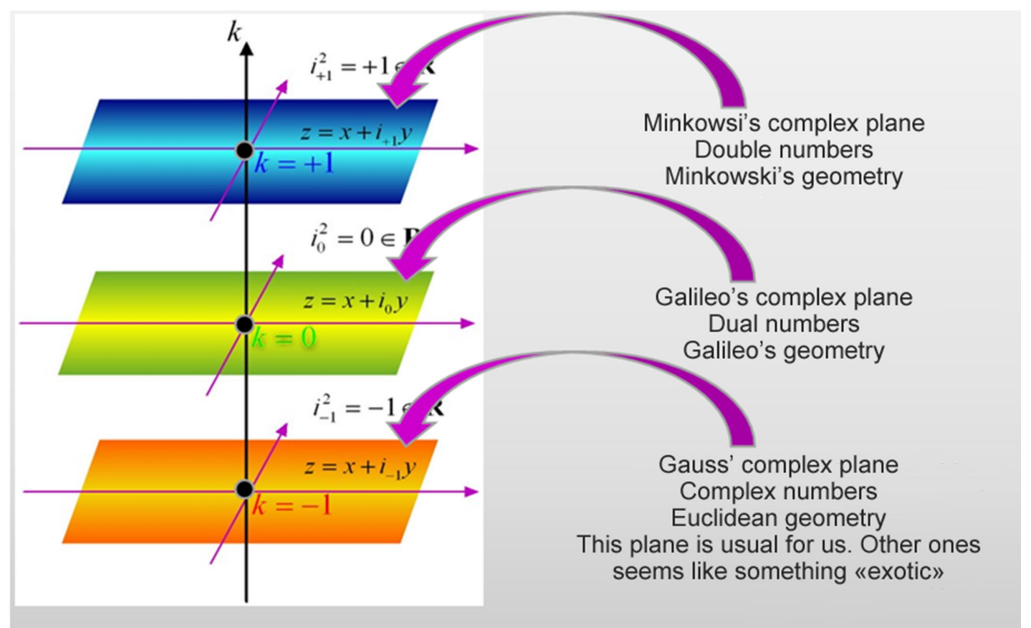

Fig. 9. In every plane, that crosses the vertical of the $k$-parameter axis, there is an algebra $\mathrm{A}_{2}\left(\mathbf{R} \mid i_{k}\right):=\left\{\mathbf{z}=a+i_{k} b \mid a, b \in \mathbf{R}\right\}$. Three planes that cross this

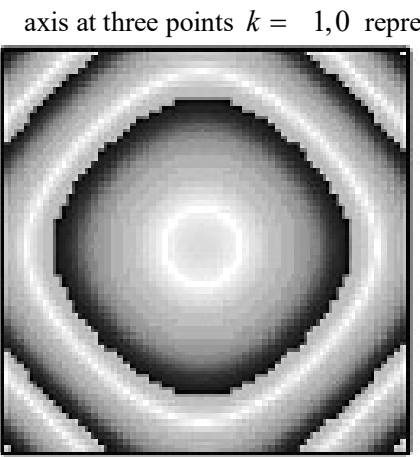

a)

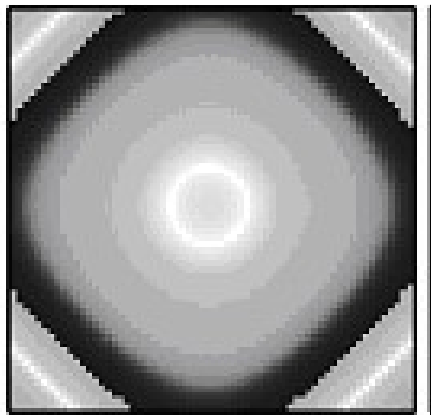

b)

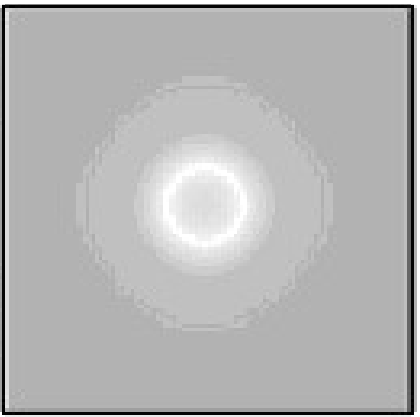

c)

Fig. 10. The excitement of three Schrödinger-Yaglom metamedia at the moment $t_{k}=128$ for three values of the parameter $k:$ a) $k=-0,25$, b)

$$
k=-0,05, \text { c) }|k| \rightarrow 0 \text { for identical values } \arg \{\mathbf{D}\}=40.5^{\circ} \text { and }|\mathbf{D}|=0.07 .
$$

\subsection{The interference of two excitements}

Because the excitement function $\varphi(x, y, t)$ frequently has a wave nature, it is very interesting to study the interference picture of two excitements that appears simultaneously in the different points of a metamedium. 
Image Processing, Geoinformation Technology and Information Security / V. Labunets et al.

Fig.11a shows us a superposition of two excitements when a diffusion coefficient is a real number. In this case both excitement processes appear to be 2-D Gaussian surfaces that add up with each other in process of time.

More interesting results can be seen on Fig. 11b-c for the Schrödinger-Euclidean metamedium with $\beta=\arg \{\mathbf{D}\}=\pi / 2, i^{2}=-1$. In that case the interference of excitements occurs, like it happens in a classical quantum mechanics. The results of an interference for the Schrodinger-Galilean metamedium with a dual diffusion coefficient $\left(i^{2}=0\right)$ are presented on Fig. 11d. Let us note that white rings of the zero phases don't add up with each other like it happens in the case of a classical interference. They are smoothly connecting instead.

\section{Conclusion}

The metamedia with a generalized complex diffusion coefficients were first studied. Their time evolutions are described with generalized Schrodinger equations. The implementation of such metamedia with a cellular automaton was considered. In addition, this work contains the results of modeling, which shown the complex character of such media's behavior. Our future work will be focused on using commutative and Clifford algebras for hyperspectral image processing and pattern recognition.

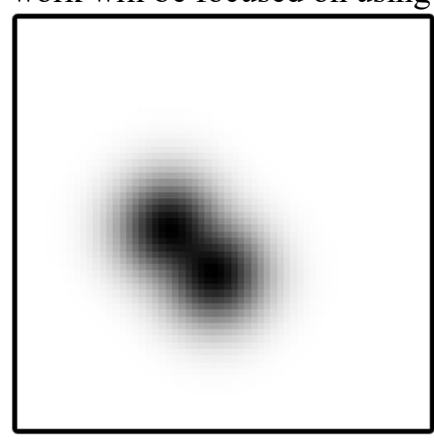

a)

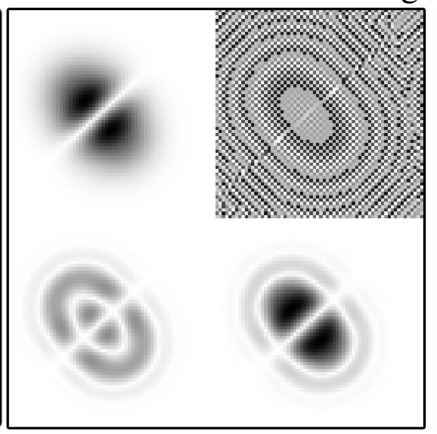

b)

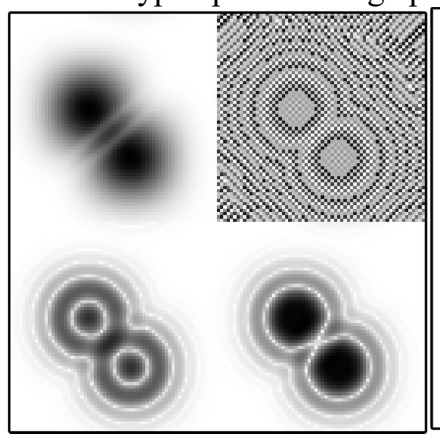

c)

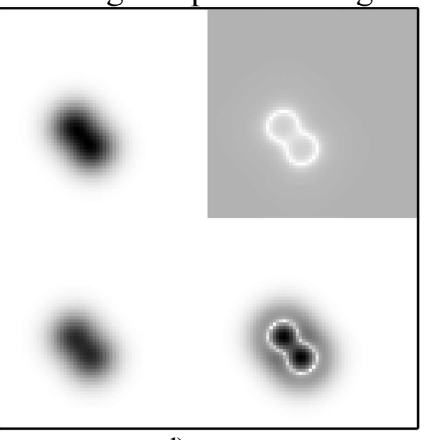

d)

Fig. 11. The interference picture of two excitements in a) the Fourier-Gaussian medium with $\beta=\arg \{D\}=0^{\circ}, i^{2}=-1$ (real diffusion coefficient); b)-c) the Schrödinger-Euclidean diffusion media (complex diffusion coefficient ): b) two closely located points were excited by the Dirac delta-functions at the initial moment of time, c) one points were located relatively far from each other, d) the interference picture of two excitements in the Schrodinger-Galilean metamedium (it has a dual diffusion coefficient).

\section{Acknowledgements}

This work was supported by grants the RFBR № 17-07-00886, № 17-29-03369 and by Ural State Forest University Engineering's Center of Excellence in "Quantum and Classical Information Technologies for Remote Sensing Systems".

\section{References}

[1] Behera L, Kar I, Elitzur A. Quantum Brain: A Recurrent Quantum Neural Network Model to Describe Eye Tracking of Moving Targets, 2000. URL: http://arxiv.org:q-bio/quant-ph/0407001v1.

[2] Nagasawa M. Schrodinger equations and diffusion theory. Monographs in mathematics. Birkheauser Verlag, Basel, Switzerland, 1993; 86: 238 p.

[3] Lou L, Zhan X, Fu Z, Ding M. Method of Boundary Extraction Based on Schrödinger Equation. Proceedings of the 21th Congress of the International Society for Photogrammetry and Remote Sensing. Beijing, China 2008; B5(2): 813-816.

[4] Hagan S, Hameroff SR, Tuzyinski JA. Quantum Computation in Brain Microtubules. Decoherence and Biological Feasibility, Physical Review E, American Physical Society 2002; 65: 1-11.

[5] Perus M, Bischof H, Caulfield J, Loo CK. Quantum Implementable Selective Reconstruction of High Resolution Images. Applied Optics 2004; 43: 61346138.

[6] Rigatos GG. Quantum Wave-Packets in Fuzzy Automata and Neural Associative Memories. International Journal of Modern Physics C, World Scientific 2007; 18(9): 209-221.

[7] Yaglom I. Complex numbers in geometry. New York.: Academic press 1968; 242: 203-205.

[8] Labunets V. Excitable Schrodinger metamedia. 23rd Internation Crimean Conference. Microwave and Telecommunication Technology. Conference proceedings 2013; I: 12-16.

[9] Wolfram S. Cellular automata as models of complexity. Reprinted from Nature. Macmillan Journals Ltd 1985; 311(5985): 419-424.

[10] Obeid I, Morizio J, Moxon K, Nicolelis MA, Wolf PD. Two Multichannel Integrated Circuits for Neural Recording and Signal Processing. IEEE Trans Biomed. Eng. 2003; 50: 255-258.

[11] Harrison R, Watkins P, Kier R, Lovejoy R, Black D, Normann R, Solzbacher F. A Low-Power Integrated Circuit for a Wireless 100-Electrode Neural Recording System. International Solid State Circuits Conference 2006; 30.

[12] Ruedi PF, Heim P, Kaess F, Grenet E, Heitger F, Burgi P-Y, Gyger S, Nussbaum P. A 128 128, pixel 120-dB dynamic-range vision-sensor chip for image contrast and orientation extraction. IEEE J. Solid-State Circuits 2003; 38: 2325-2333.

[13] Lichtsteiner P, Posch C, Delbruck T. A 128128120 dB 30mW asynchronous vision sensor that responds to relative intensity change. IEEE J. Solid-State Circuits 2008; 43: 566-576. 